\title{
Et la linguistique au Brésil
}

Le rôle d'Émile Benveniste

Valdir do Nascimento Flores

\section{(2) OpenEdition \\ Journals}

Édition électronique

URL : http://journals.openedition.org/aes/468

DOI : $10.4000 /$ aes.468

ISSN : 2258-093X

Éditeur

Laboratoire LISAA

Référence électronique

Valdir do Nascimento Flores, « Et la linguistique au Brésil », Arts et Savoirs [En ligne], 2 | 2012, mis en ligne le 15 juillet 2012, consulté le 04 mai 2019. URL : http://journals.openedition.org/aes/468 ; DOI : $10.4000 / a e s .468$

Ce document a été généré automatiquement le 4 mai 2019.

Centre de recherche LISAA (Littératures SAvoirs et Arts) 


\title{
Et la linguistique au Brésil
}

\author{
Le rôle d'Émile Benveniste
}

\author{
Valdir do Nascimento Flores
}

Ce texte est le résultat de la session de la Conférence plénière donnée au colloque "Les Théories de l'énonciation aujourd'hui ; Benveniste après un demi-siècle ». J'aimerais remercier le comité organisateur pour l'invitation à participer de ce colloque.

1 Ce texte est divisé en deux parties : dans la première partie, je vais retracer l'histoire de la réception de la théorie énonciative d'Émile Benveniste par la linguistique brésilienne. L'objectif est donc de répondre à la question suivante: quelle lecture la linguistique brésilienne a-t-elle fait de la théorie énonciative de Benveniste? Dans la deuxième partie, je présenterai certains opérateurs de lecture de la théorie de Benveniste qui sont, à mon avis, responsables d'une nouvelle interprétation de la théorie dans le contexte de la linguistique brésilienne.

\section{Première partie}

2 La linguistique brésilienne a commencé à étudier les théories énonciatives à la fin des années soixante-dix. Néanmoins, c'est dans les années quatre-vingt que les linguistes brésiliens ont réellement utilisé les théories de l'énonciation pour étudier des phénomènes linguistiques de la langue portugaise. Dans ce sens, la présence de l'énonciation au Brésil est très récente.

3 Les spécificités de cette présence découlent de la manière dont la linguistique brésilienne a abordé le champ énonciatif à cette époque. Au Brésil, les théories de l'énonciation ont en effet été l'objet d'une lecture très particulière. Certaines ont été identifiées à la pragmatique, d'autres à l'analyse du discours et d'autres encore à la linguistique textuelle. À travers cette observation, j'aimerais mettre l'accent sur le fait que l'apparition du champ de l'énonciation au Brésil a en quelque sorte eu lieu à travers d'autres disciplines des études du langage. On peut donc dire qu'il y a eu une sorte 
d'« utilisation » de l'apparat méthodologique des théories énonciatives sans l'incorporation de l'épistémologie sous-jacente aux modèles.

Dans les années 1980 et même 1990, les linguistes brésiliens ont utilisé les théories de l'énonciation en ne tenant compte que du potentiel descriptif des modèles, sans se soucier de leur fondement épistémologique. La lecture de la théorie énonciative d'Émile Benveniste en est un parfait exemple. Personnellement, je pense qu'au Brésil Benveniste n'a pas été lu avec l'intention de comprendre la complexité de sa pensée sur le langage, mais seulement pour servir de support à d'autres théories. Pendant les années 1980, sa théorie a davantage servi de soutien à la pragmatique, à l'analyse du discours et à la linguistique textuelle. Pour illustrer mes propos, je vais citer quelques exemples.

Dans le champ de la pragmatique, les chercheurs citent normalement le texte La nature des pronoms et se contentent de voir dans les pronoms une marque de la deixis. Ils oublient de les associer à la distinction - très importante - opérée par l'auteur entre personne/ non-personne. Les linguistes de la pragmatique oublient que Benveniste s'est basé sur la classe des pronoms pour évoquer une notion qui va bien au-delà. En effet, il parle de quelque chose qui est « un problème de langage et un problème de langues, ou mieux, qu'il n'est un problème de langues que parce qu'il est d'abord un problème de langage $»^{1}$. À travers les pronoms, il parle ainsi de l'universalité de la présence de l'homme dans la langue.

6 Les chercheurs de l'analyse du discours citent également Benveniste, mais dans le sens d'une critique des notions de sujet et de subjectivité. Au Brésil, l'analyse du discours fait penser d'une part que Benveniste a introduit la réflexion sur le sujet dans les études du langage, mais d'autre part elle critique cette notion. De l'avis des analystes du discours brésiliens, le sujet de Benveniste possède une nature psychologique, égocentrique et idéaliste. Pour étayer leurs critiques, les analystes du discours se fondent plus particulièrement sur les textes «Les relations de temps dans le verbe français ", "De la subjectivité dans le langage » et «L'appareil formel de l'énonciation ». Dans des extraits "De la subjectivité dans le langage», ils trouvent la preuve de la perspective égocentrique de Benveniste; c'est notamment le cas du passage "Est "ego" qui dit “ego" " ${ }^{2}$. Dans «Les relations de temps dans le verbe français ", l'objet de la critique de l'analyse du discours est la distinction entre énonciation historique et énonciation de discours. Et dans «L'appareil formel de l'énonciation », la critique s'effectue sur l'idée d' appropriation.

7 Il est important de rappeler qu'à cette époque, au Brésil, l'analyse du discours se basait fortement sur une vision marxiste des relations de sens dans le langage. Conséquemment, la critique était très liée au champ du politique. Il en est de même pour les chercheurs de la linguistique textuelle brésilienne; là encore, ils n'ont pas cherché à comprendre la pensée de Benveniste mais se sont contentés d'utiliser les études de l'énonciation pour appuyer les descriptions du texte. Dans le champ de la linguistique textuelle, on ne trouve que quelques références aux études sur le verbe et le pronom, utilisées pour décrire des marques de temporalité, de cohésion textuelle, de modalités, etc.

8 Je pense que la présence de Benveniste dans la linguistique textuelle est similaire à celle observée dans les études pragmatiques: l'accent est mis sur l'aspect descriptif de la théorie au détriment de la réflexion qui la sous-tend.

9 En résumé, et pour conclure la première partie de cet exposé, il me semble important de souligner que la théorie énonciative de Benveniste jouit assurément d'une certaine 
reconnaissance dans la linguistique brésilienne. Mais que cette reconnaissance est soit accompagnée de critiques - c'est le cas de l'interprétation égocentrique du sujet faite à travers l'analyse du discours -, soit accompagnée d'une approche un tant soit peu superficielle - c'est le cas de la pragmatique et de la linguistique textuelle. On peut donc en déduire dans un premier temps que la linguistique brésilienne a attribué une certaine importance à la réflexion benvenistienne mais sans l'évaluer de manière adéquate, parce qu'elle l'a associée à un autre champ théorique (pragmatique, textuel ou discursif) ou n'a pas tenté d'en comprendre la complexité. En d'autres termes, la manière dont la linguistique brésilienne s'est approprié le système conceptuel de Benveniste a chaque fois donné lieu à une configuration épistémologique différente de sa configuration originale. Ainsi, l'apparition des études énonciatives dans le contexte brésilien - du moins tel que je l'ai présenté jusqu'à maintenant - a été relativement compliquée. Mais cela n'a pas empêché les spécialistes de s'intéresser, même indirectement, au champ de l'énonciation.

\section{Deuxième partie}

Jusqu'à présent, j'ai fait une sorte de bilan sur la présence de Benveniste dans la linguistique brésilienne. Dans cette deuxième partie, je propose de voir comment a été envisagée la théorie énonciative de Benveniste au Brésil au cours des dernières années. Cette lecture s'attache avant tout à examiner le système conceptuel de l'auteur. Il ne s'agit plus de comparer Benveniste avec d'autres champs de la linguistique, mais plutôt de comprendre la réflexion de l'auteur.

11 À ce stade, la question qui oriente mon travail est la suivante : comment est-il possible de relire Benveniste?

12 Mais avant d'aborder cette nouvelle lecture, une remarque est nécessaire: ce qui suit reste très modeste et ne concerne que ma propre manière de lire Benveniste, une approche bâtie sur plus de vingt années consacrées à l'enseignement de la linguistique benvenistienne. Mon expérience découle de cet enseignement ainsi que de la production d'un certain nombre de matériels de référence (dictionnaires, manuels d'introduction, thèses et mémoires universitaires). Je ne parle donc pas au nom des linguistes brésiliens, d'autant qu'il n'existe pas au Brésil de point de vue reflétant l'opinion d'un ensemble de linguistes sur la linguistique énonciative de Benveniste. À l'exemple de ce que dit Benveniste dans La forme et le sens dans le langage, «celui qui parle ici le fait en son nom personnel et propose des vues qui lui sont propres $»^{3}$.

13 Je vais présenter une liste de points que je considère très importants pour relire Benveniste.

14 Premier point : l'œuvre de Benveniste dépasse le champ de l'énonciation. Si le nom de Benveniste est surtout prononcé dans des discussions liées au champ des études du discours, au sens large, il serait cependant injuste et incorrect d'ignorer que sa réflexion transcende ce que l'on entend aujourd'hui sous l'appellation «études du discours». Je pense donc qu'étudier Benveniste oblige à préciser la partie de l'œuvre analysée, car l'œuvre dans son ensemble dépasse le champ de l'énonciation. Étudier cette œuvre implique de faire des recoupements et de constituer un corpus textuel de référence à partir duquel peut être développée une recherche. Autrement dit, il y a une œuvre benvenistienne. Sur l'ensemble de l'œuvre envisagé comme un corpus initial formé de sources de différentes natures, il faut recouper, sur la base d'objectifs spécifiques, un 
corpus textuel de recherche. Benveniste a produit une pensée tout à fait singulière, dont la complexité ne pourrait être saisie que par une étude épistémologique exhaustive. Son œuvre analyse une infinité de thèmes : linguistique générale, phénomènes diachroniques, syntaxiques, lexicaux, culturels, etc. Parallèlement, certains thèmes sont nettement abordés en interaction avec des domaines connexes aux études du langage (philosophie, psychanalyse, sociologie, anthropologie, théories de la culture, de la logique, etc.). Les sources d'Émile Benveniste sont nombreuses, et à travers elles il parle de la phonologie, de la syntaxe, de la sémantique, de la morphologie, de la pragmatique ainsi que de plusieurs autres niveaux de l'analyse linguistique et son rapport avec d'autres champs. Par conséquent, une conclusion s'impose : il est nécessaire de choisir un point de vue à partir duquel il devient possible de sélectionner le corpus textuel de recherche. En ce qui me concerne, j'aimerais présenter ici une possibilité de compréhension de ce que l'on a convenu d'appeler la théorie de l'énonciation de Benveniste. Pour y parvenir, le corpus à la base de mes observations est constitué d'un ensemble de textes réunis dans Problèmes de linguistique générale I et II.

Deuxième point : il faut instaurer un point de vue de lecture. Nous partons d'un principe épistémologique de lecture: la théorie de Benveniste peut être lue comme un réseau complexe dont les termes et les notions sont reliés entre eux à partir de différentes relations - hiérarchiques, parallèles, transversales, etc. Dans ce sens, nombre des concepts proposés par Benveniste ont une valeur primitive puisqu'ils intègrent d'autres concepts. Autrement dit, les termes et les notions faisant partie d'un concept donné contiennent d'autres termes et d'autres notions, eux-mêmes contenus dans plusieurs autres. Prenons par exemple une réflexion de Benveniste : «La "subjectivité" dont nous traitons ici est la capacité du locuteur à se poser comme "sujet" $»^{4}$. Face à cette formulation, comprendre ce qu'est la « subjectivité » exige de comprendre ce que l'auteur entend par « locuteur » et par « sujet », deux notions qui renvoient à leur tour à d'autres notions (« langue » et « langage » par exemple). Si l'on admet ce raisonnement, on peut alors dire que la compréhension de certains concepts, termes et notions chez Benveniste résulte des relations qu'ils entretiennent avec d'autres concepts, termes et notions. Cela revient à dire qu'il est difficile d'étudier un élément de manière isolé dans cette théorie. Notre lecture de la théorie énonciative de Benveniste est par conséquent la suivante: considérer cette théorie comme un réseau de primitifs théoriques. Certes, les relations varient dans la mesure où les termes qui servent de points de départ varient également. On pourrait même aller jusqu'à interroger la pertinence de ce qui est présenté comme terme intégrant Mais là n'est pas la question : nous soutenons l'existence des relations sans affirmer pour autant qu'elles se configurent sous une forme ou une autre. Pour l'instant, accepter ou non quel terme intègre chaque ensemble de relations est secondaire. L'essentiel est de percevoir que la réflexion benvenistienne ne peut être lue de façon linéaire.

Troisième point : il est possible de travailler avec des « moments thématiques ». Je pense qu'il est possible de dire que la réflexion sur l'énonciation développée pendant quarante ans par Benveniste admet une division thématique, et ce même si cette division ne coïncide pas nécessairement avec la chronologie des textes ou avec ce qui pourrait être nommé les phases de la théorie. Je vais donner un exemple. Prenons la célèbre distinction personne/non-personne. Si cette distinction est très fréquente dans les articles sur l'étude du verbe et du pronom produits dans les années quarante et cinquante, elle l'est un peu moins dans les textes qui ont suivi. Une consultation rapide des textes présents 
dans les deux tomes de Problèmes de linguistique générale permet de voir que le terme nonpersonne apparaît seulement dans : "Structure des relations de personne dans le verbe ", de 1946, «La nature des pronoms », de 1956, « De la subjectivité dans le langage », de 1958 et dans «Structure de la langue, Les relations de temps dans le verbe français », de 1959, et «Structure de la société », de 1968. Le terme non-personne n'est utilisé que dans un seul texte des années 1960, à savoir "Structure de la langue et structure de la société ». Il s'agit là sans aucun doute d'un indicateur important. Le chercheur peut alors se demander : dans la mesure où il progresse dans sa recherche sur l'énonciation, pourquoi Benveniste met-il moins l'accent sur la dichotomie personne/non-personne? Cette question est d'autant plus importante si l'on observe que des notions majeures telles que la sémiotique et la sémantique sont élaborées dans les textes des années soixante, et celle de l'appareil formel de l'énonciation dans les années 1970. Je pense donc qu'il est possible de parler de l'existence de noyaux thématiques autour desquels sont associés des notions, des termes et des concepts. Par exemple, autour de la distinction personne/non-personne nombre de concepts, notions et termes sont présents: corrélation de personnalité, corrélation de subjectivité, je, tu, il, énonciation de discours, énonciation historique, temps linguistique, signes vides, signes pleins, unicité, sujet, locuteurs, homme, subjectivité, intersubjectivité, indicateurs auto-référentiels, indicateurs de subjectivité, indicateurs de la deixis, instance de discours, instance d'énonciation, situation de discours, énonciations, et bien d'autres encore. Autour de la distinction sémiotique/ sémantique, on rencontre notamment : forme, sens, phrase, syntagmation, sémantisation, être reconnu, être compris, mot, actualisation, langue-discours, discours, référence, énoncée, énonciation, analyse intra-linguistique, analyse translinguistique, métasémantique ou encore sémiologie de deuxième génération. Si le noyau thématique personne/non-personne n'est pas limité à une période spécifique de temps (on trouve des textes des années 1940, 1950 et 1960), les textes sur le couple sémiotique/sémantique sont au contraire restreints aux années 1960, à savoir : "La forme et le sens dans le langage » (1966/1967) et « Sémiologie de la langue » (1969). Un troisième exemple peut encore être donné : quand on considère la notion d'appareil formel de l'énonciation, on s'aperçoit qu'elle est aussi entourée par d'autres termes : appareil formel de la langue, appropriation, réalisation vocale de la langue, cadres figuratifs, dialogue, indices spécifiques, procédés accessoires, énonciation, entre autres.

17 J'aimerais ici faire une observation: de mon point de vue, le moment où Benveniste élabore la réflexion sur l'appareil formel de l'énonciation constitue un moment synthèse de l'œuvre, qui contient tous les autres et oriente en quelque sorte la recherche vers la linguistique énonciative. Comme il le dit d'ailleurs lui-même à la fin de L'appareil formel de l'énonciation, «de longues perspectives s'ouvrent à l'analyse des formes complexes du discours, à partir du cadre formel esquissé ici $»^{5}$.

Bien évidemment, cette interprétation que je fais de l'ensemble des textes écrits par Benveniste sur l'énonciation ne peut être confondue avec la mise en œuvre d'un type de phase de la théorie. Je ne dis pas qu'il y a des phases dans sa théorie, vu que les concepts développés à certains moments sont généralement repris, amplifiés et approfondis à un autre moment. Je crois simplement que la théorie énonciative benvenistienne dans son ensemble peut être lue à partir de la prise en compte de moments thématiques. D'autre part, je pense que chacun d'eux présente des possibilités distinctes de théorisation et d'analyse des faits linguistiques. 
Quatrième point : il n'existe pas une seule manière d'étudier l'énonciation. Benveniste dit qu'il y a des aspects de l'énonciation. Dans L'appareil formel de l'énonciation, il écrit : « Ce grand procès peut être étudié sous divers aspects. Nous en voyons principalement trois ${ }^{6}$ . Le premier aspect est le vocal : «Le plus immédiatement perceptible et le plus direct bien qu'en général on ne le mette pas en rapport avec le phénomène général de l'énonciation - est la réalisation vocale de la langue. ${ }^{7}$ Je pense que beaucoup de recherches peuvent encore être faites pour développer cet aspect de l'énonciation qui, à mon avis, est seulement présenté de façon programmatique par Benveniste. Le deuxième aspect est celui de la sémantisation. D'après l'auteur :

Le mécanisme de cette production est un autre aspect majeur du même problème. L'énonciation suppose la conversion individuelle de la langue en discours. Ici la question - très difficile et peu étudiée encore - est de voir comment le «sens » se forme en "mots », dans quelle mesure on peut distinguer entre les deux notions et dans quels termes décrire leur interaction. C'est la sémantisation de la langue qui est au centre de cet aspect de l'énonciation, et elle conduit à la théorie du signe et à l'analyse de la signifiance. ${ }^{8}$

On le voit, dans cet extrait Benveniste insiste sur l'aspect opératoire de l'énonciation. Il ajoute d'ailleurs que «sous la même considération nous rangerons les procédés par lesquels les formes linguistiques de l'énonciation se diversifient et s'engendrent ${ }^{9}$. Quant au troisième aspect, c'est celui du cadre formel de réalisation : Benveniste signale qu'« on peut enfin envisager une autre approche, qui consisterait à définir l'énonciation dans le cadre formel de sa réalisation. $»^{10}$

21 Il me semble que ces exemples sont suffisants pour illustrer la thèse selon laquelle l'énonciation peut être vue sous différents points de vue dans la théorie de Benveniste.

Cinquième point : la théorie énonciative de Benveniste n'est pas un modèle achevé. Je tiens tout d'abord à rappeler que l'expression théorie de l'énonciation n'est pas utilisée par Benveniste. Et si ma lecture des Problèmes de linguistique générale est bonne, ce syntagme n'apparaît dans aucun de ses articles. Ce qui se rapproche le plus du syntagme théorie de l'énonciation, c'est l'expression sémantique de l'énonciation présente à la fin de «La sémiologie de la langue $»^{11}$. On peut même dire que ladite théorie de l'énonciation de Benveniste est davantage une déduction faite a posteriori par les lecteurs des articles réunis dans Problèmes de linguistique générale I et II qu'une intention explicite de l'auteur.

Partant de là, je pense que l'on ne peut pas attribuer à Benveniste la démarche délibérée de produire une théorie, celle de l'énonciation. Rien n'indique qu'il a eu l'intention d'écrire une théorie : à l'inverse de ce que l'on peut voir aujourd'hui chez d'autres auteurs, aucun de ses témoignages ne nous autorise à penser qu'il avait comme objectif d'élaborer une théorie achevée. Donc la désignation théorie de l'énonciation pour le travail de Benveniste sur l'énonciation ne doit pas être entendue dans le sens d'un modèle achevé, ni même d'un modèle en construction. Dans ce contexte, le mot théorie n'a pas le même sens que celui présent par exemple dans le syntagme théorie générative. Comprendre cela quand on lit Benveniste est extrêmement important, parce que cet aspect place la réflexion de l'auteur dans une position très singulière au sein de la linguistique. De plus, Benveniste ne propose explicitement aucun modèle de base pour toutes ses analyses. Par conséquent, chaque texte possède sa propre logique. Ils ne peuvent pas être vus comme un ensemble congruent de propositions théoriques et méthodologiques. Au contraire, chaque texte propose des catégories particulières d'analyse, avec des théorisations et des 
développements spécifiques à ces analyses. Chaque texte renferme en soi des types spécifiques d'analyse des langues, de la langue et du langage.

D'où le constat suivant: les textes qui parlent d'énonciation ne sont pas comparables entre eux. C'est pourquoi on ne peut s'empêcher d'être étonnés quand on voit, en général dans des travaux universitaires, le passage d'un texte à l'autre comme s'ils traitaient du même sujet. Cet aplanissement des différences existantes entre les textes atteste à mon avis de la méconnaissance de l'ensemble duquel ils font partie. En d'autres mots, je soutiens l'idée selon laquelle il y a dans chaque texte, pris dans sa logique particulière, des potentialités théoriques et analytiques spécifiques. De tels textes peuvent à la limite être regroupés sous un même grand moment thématique, mais sans ignorer leur logique particulière. Cela expliquerait, en principe du moins, le grand flottement conceptuel et terminologique qu'il y a entre les textes.

Sixième point : on ne peut ignorer la diachronie de la pensée de Benveniste. Si chaque texte possède sa propre logique, alors je pense qu'il n'est pas possible de lire synchroniquement ce qui a été écrit en diachronie : lire les textes de Benveniste comme s'ils étaient contemporains les uns des autres reviendrait à ne pas respecter leur chronologie, au demeurant fondamentale. Benveniste a opéré des déplacements dans son travail. Et suivre ces déplacements, c'est dessiner la diachronie d'une pensée en formation. Je vais donner ici un exemple qui illustre l'importance de prendre en compte la diachronie de la pensée de Benveniste : le lecteur qui étudie les textes de Benveniste écrits au début des années quarante - à l'exemple du texte "Structure des relations de personne dans le verbe » (1946) - va percevoir que la notion d'énonciation qui y apparait n'est pas la même que celle rencontrée en 1970 dans "L'appareil formel de l'énonciation ». Dans "L'appareil formel de l'énonciation", on rencontre la célèbre définition de l'énonciation : «L'énonciation est cette mise en fonctionnement de la langue par un acte individuel d'utilisation $»^{12}$. Hormis les nombreux dédoublements de cette notion tout au long du texte, il est facile de voir que l'énonciation dont il est ici question est traitée du point de vue d'un acte exécuté par le locuteur. Cela apparaît textuellement dans la définition. Dans «Les relations de temps dans le verbe français ", de 1959, soit près d'une décennie avant "L'appareil formel de l'énonciation", l'énonciation est évoquée pour effectuer une distinction très importante. Benveniste écrit :

Les temps d'un verbe français ne s'emploient pas comme les membres d'un système unique, ils se distribuent en deux systèmes distincts et complémentaires. Chacun d'eux ne comprend qu'une partie des temps du verbe; tous les deux sont en usage concurrent et demeurent disponibles pour chaque locuteur. Ces deux systèmes manifestent deux plans d'énonciation différents, que nous distinguerons comme celui de l'histoire et celui du discours. ${ }^{13}$

Dans cet extrait, j'attire l'attention sur le fait que Benveniste parle de "plans d'énonciation ». Pour lui, il a ainsi deux plans d'énonciation. Les deux plans d'énonciation se délimitent donc en traits positifs et négatifs :

- dans l'énonciation historique, sont admis (en forme de troisième personne) : l'aoriste, l'imparfait, le plus-que-parfait et le prospectif ; sont exclus : le présent, le parfait, le futur (simple et composé) ;

- dans l'énonciation de discours, sont admis tous les temps à toutes les formes; est exclu l'aoriste (simple et composé). ${ }^{14}$

Comme on peut le voir, Benveniste établit dans Les relations de temps dans le verbe français une différence à caractère formel, avec l'inclusion ou non de l'aoriste. En conséquence, le 
mot énonciation dans le syntagme énonciation historique serait beaucoup mieux compris s'il était pris dans le sens d'énoncé, c'est-à-dire de produit de la parole. Idem pour le terme énonciation présent dans le syntagme énonciation de discours. En 1959, la différence entre histoire et discours est de nature formelle; elle est la marque de l'énoncé qui est le trait différenciateur de l'une et de l'autre. Il ne serait donc pas déraisonnable de penser qu'en parlant d'énonciation historique et d'énonciation de discours Benveniste soit en fait en train de parler de type d'énoncés. Dans "Les relations de temps dans le verbe français", l'utilisation du terme énonciation ne se réfère pas à l'acte d'énoncé, il a donc un sens différent de celui présent dans "L'appareil formel de l'énonciation ».

Septième point: il y a une fluctuation terminologique dans la théorie énonciative de Benveniste. Il y a une fluctuation terminologique chez Benveniste. Dans sa théorie, il est très facile de rencontrer des homonymies et des synonymies terminologiques. Je vais donner quelques exemples.

Il y a homonymie à l'intérieur d'un même texte. Je vais prendre comme exemple l'utilisation du terme sens dans le texte Les niveaux de l'analyse linguistique (1962/1964). Ce terme y apparaît plus de trente fois, mais je ne vais en analyser que deux. L'une de ces occurrences apparaît dans une structure définitionnelle du type $X$ est $Y$, ce qui est très rare chez Benveniste. Il écrit: "Le sens d'une unité linguistique se définit comme sa capacité d'intégrer une unité de niveau supérieur $\aleph^{15}$. Mais aussitôt après, il souligne : «mais la notion de sens a encore un autre aspect $»^{16}$. Et il ajoute finalement: « un tout autre problème serait de demander: quel est ce sens? Ici "sens » est pris en une acception complètement différente $»^{17}$. Grosso modo, Benveniste établit dans ce texte au moins deux «sens " pour le terme sens. Du point de vue terminologique, il y a entre ces deux emplois de sens une relation d'homonymie terminologique.

Il y a homonymie dans des textes différents. Je prends comme exemple l'utilisation du terme énonciation dans deux textes chronologiquement très proches l'un de l'autre : « La phrase nominale » (1950) et « De la subjectivité dans le langage » (1958). Dans La phrase nominale, on peut lire : «Étant apte à des assertions absolues, la phrase nominale à valeur d'argument, de preuve, de référence. [...] C'est pourquoi la phrase nominale convient si bien à ces énonciations où elle tend d'ailleurs à se confiner, sentences ou proverbes, après avoir connu plus de souplesse. $»^{18} \mathrm{Et}$ dans De la subjectivité dans le langage, il est écrit : « En disant je promets, je garantis, je promets et je garantis effectivement. Les conséquences (sociales, juridiques, etc.) de mon jurement, de ma promesse, se déroulent à partir de l'instance de discours contenant je jure, je promets. L'énonciation s'identifie avec l'acte même. $»^{19}$ Le simple fait de mettre en regard ces deux emplois d'énonciation éclaircit la différence. Dans le premier texte, énonciations écrit au pluriel se réfère à des assertions absolues. Dans le deuxième, l'énonciation utilisée au singulier a un sens beaucoup plus proche de celui qu'on a l'habitude d'attribuer à Benveniste dans le champ énonciatif. Ici, énonciation est vue comme un acte.

En réalité ce sont des termes homonymes, qui confirment la thèse selon laquelle la lecture des textes de Benveniste doit respecter la logique interne de chacun d'eux. Il y a synonymie dans un même texte. À titre d'exemple, on peut comparer ici l'utilisation des termes instance de discours et situation de discours dans le texte « La nature des pronoms »(1956). Observons deux passages :

(1) je ne peux être identifié que par l'instance de discours qui le contient et par là seulement. ${ }^{20}$ 
(2) C'est en s'identifiant comme personne unique prononçant je que chacun des locuteurs se pose tour à tour comme «sujet». L'emploi a donc pour condition la situation de discours et nulle autre. ${ }^{21}$

Si Benveniste parle de la présence de l'homme dans la langue, sa linguistique va finalement très au-delà de ce que nous sommes en mesure d'évaluer. Comme il le dit luimême à la fin de "L'appareil formel de l'énonciation ", et que j'ai déjà eu l'occasion de citer au début de ce texte, «des longues perspectives s'ouvrent à l'analyse des formes complexes du discours, à partir du cadre formel esquissé ici ».

Dans cette utilisation de situation de discours, il est possible d'apercevoir les mêmes caractéristiques que celles d'instance de discours. Autrement dit, ce sont des termes synonymes au sein d'un même texte.

Je pourrais certes donner beaucoup d'autres exemples. Néanmoins, je pense que les exemples déjà donnés sont suffisants pour montrer combien il est important de respecter la logique interne de chaque texte et la diachronie de la pensée de Benveniste.

\section{Conclusion : il y a un principe unificateur de la théorie}

En guise de conclusion, j'aimerais rappeler qu'il y a un principe qui traverse toute la réflexion de Benveniste sur le langage. Je ne vais pas m'y étendre parce que cela dépasserait le cadre de cette présentation, cependant il me semble bon d'observer que ce principe est commun y compris aux études de Benveniste qui se situent en dehors de ladite théorie de l'énonciation. Ce principe qui traverse toute la réflexion de Benveniste est le suivant: l'homme est dans la langue. "L'homme dans la langue ", le titre de la cinquième partie des Problèmes de linguistique générale.

$\mathrm{Au}$ cours des dernières années, je me suis consacré à l'étude de ces formes complexes du permettant à l'homme d'être dans la langue, à l'homme de se singulariser, de devenir sujet. discours. À mon avis, ces formes complexes représentent les nombreuses possibilités Le principe épistémologique est unique et explicitement présent dans la théorie depuis le texte «De la subjectivité dans le langage », de 1958; et il est récurrent dans tous les autres textes, y compris dans ceux qui ne traitent pas directement de l'énonciation. Pour terminer, je cède la parole à Benveniste :

Nous n'atteignons jamais l'homme séparé du langage et nous ne le voyons jamais l'inventant. Nous n'atteignons jamais l'homme réduit à lui-même et s'ingéniant à concevoir l'existence de l'autre. C'est un homme parlant que nous trouvons dans le monde, un homme parlant à un autre homme, et le langage enseigne la définition même de l'homme. ${ }^{22}$

\section{BIBLIOGRAPHIE}

Benveniste, Émile, « De la subjectivité dans le langage » [1958], Problèmes de linguistique générale, Tome 1, Paris, Gallimard, 1966. 
Benveniste, Émile, « La nature des pronoms » [1956], Problèmes de linguistique générale, Tome 1, Paris, Gallimard, 1966.

Benveniste, Émile, « La phrase nominale » [1950], Problèmes de linguistique générale, Tome 1, Paris, Gallimard, 1966.

Benveniste, Émile, « Les niveaux de l'analyse linguistique » [1964], Problèmes de linguistique générale, Tome 1, Paris, Gallimard, 1966.

Benveniste, Émile, « Les relations de temps dans le verbe français » [1959], Problèmes de linguistique générale, Tome 1, Paris, Gallimard.

Benveniste, Émile, «Structure des relations de personne dans le verbe » [1946], Problèmes de linguistique générale, Tome 1, Paris, Gallimard.

Benveniste, Émile, « Sémiologie de la langue » [1969], Problèmes de linguistique générale, Tome 2, Paris, Gallimard, 1974.

Benveniste, Émile, «L'appareil formel de l'énonciation » [1970], Problèmes de linguistique générale, Tome 2, Paris, Gallimard, 1974.

Benveniste, Émile, « La forme et le sens dans le langage » [1967], Problèmes de linguistique générale, Tome 2, Paris, Gallimard, 1974.

\section{NOTES}

1. Émile Benveniste, "La nature des pronoms", Problèmes de linguistique générale, 1. Paris, Gallimard, 1966, p. 251.

2. Émile Benveniste, "de la subjectivité dans le langage ", Problèmes de linguistique générale, 2. Paris, Gallimard, 1974, p. 260.

3. Émile Benveniste, « La forme et le sens dans le langage », ibid.., p. 217.

4. Émile Benveniste, « de la subjectivité dans le langage », ibid., p. 259.

5. Émile Benveniste, « L'appareil formel de l'énonciation », ibid., p. 88.

6. Ibid., p. 80.

7. Ibid., p. 81.

8. Ibid.

9. Ibid.

10. Ibid.

11. Ibid., p. 66.

12. Ibid. p. 80.

13. Émile Benveniste, «Les relations de temps dans le verbe français », op. cit., p. 238.

14. Ibid, p. 245.

15. Ibid., p. 127.

16. Ibid.

17. Ibid., p. 127.

18. Ibid., p. 165.

19. Émile Benveniste, « De la subjectivité dans le langage », op. cit., p. 265.

20. Op. cit., p. 252.

21. Ibid., p. 254.

22. Op. cit., p. 259. 
INDEX

Mots-clés : linguistique, Brésil, discours

\section{AUTEUR}

VALDIR DO NASCIMENTO FLORES 